\title{
Effects of Natural Light Dilution on Microalgae Growth
}

\author{
Irina Harun, Liyana Yahya, Muhammad Nazry Chik, Nur Nadia Abdul Kadir, and Mohd Asyraf Mohd \\ Azmir Pang
}

\begin{abstract}
In this study, the growth of the microalgae, Isochrysis sp. under different illumination levels of natural sunlight was evaluated. Isochrysis sp. was cultured in $\mathbf{f} / \mathbf{2}$ media with five variations of illumination exposures. Each experimental setup was cultured in a $1 \mathrm{~L}$ cylindrical column with aeration provided by an air pump. Results showed that the best growth was exhibited when microalgae culture was exposed to $73.6 \%$ of illumination exposure with a light-dark cycle of 43.88-28.36s per height of column. Said culture also gave the highest $\mathrm{CO}_{2}$ fixation rate of $0.541 \mathrm{~g} \mathrm{CO}_{2} / \mathrm{day}$. This proved that optimum illumination exposure is one of the most important factors towards improving microalgae growth rate and enhancing its' carbon fixation ability.
\end{abstract}

Index Terms - Carbon fixation rate, illumination, Isochrysis sp., light-dark cycle.

\section{INTRODUCTION}

Historically, the Earth's climate has been constantly changing which accounts for the variability of life that evolves to adapt to the environment. These changes however, were interspaced thousands of years between each other. But in recent years, the rate of climate change has been quite erratic and causes concerns of the adverse effects global warming has on the Earth's stability. Carbon dioxide is an important greenhouse gas but human activities such as utilization of fossil fuels, agriculture, and deforestation have altered the $\mathrm{CO}_{2}$ concentration in the Earth's atmosphere and contributed to the global warming problems [1]. This highlights the necessity of carbon capture and storage technologies as one of the approaches to counter the environmental concerns rising from global warming [2].

In terms of carbon capture and storage, various studies have been done to showcase the ability of carbon fixation by microalgae, which showed better results compared to plants due to its higher growth rate [3]. After all, both microalgae and plants capture and fix $\mathrm{CO}_{2}$ through natural photosynthesis process. Therefore, the higher growth rate of microalgae indicates that the rate of photosynthesis, and subsequently $\mathrm{CO}_{2}$ fixation rate is much higher for microalgae compared to plants. This process, which essentially employs carbon recycling, is in fact the prime method employed by nature in carbon management. The method is so intelligently

Manuscript received September 10, 2013; revised November 13, 2013 This work was supported by TNB Research Sdn. Bhd., Malaysia through the TNB Research Fund.

Irina Harun, Liyana Yahya, Muhammad Nazry Chik, and Mohd Asyraf Mohd Azmir Pang are with the TNB Research Sdn Bhd, Kajang 43000, Malaysia (tel.: +60389225000; fax: +60389268828; e-mail: irina@tnbr.com.my, mnazry.chik@tnbr.com.my,masyraf.mapang@tnbr.com.my).

Nur Nadia Abdul Kadir is with the Chemical Engineering Department, Universiti Sains Malaysia, 14300 Nibong Tebal, Malaysia (e-mail: nurnadia_nad96@rocketmail.com). designed and executed in perfect synergy and balance, among the carbon network 'players', that physical storage of $\mathrm{CO}_{2}$ is minimal or deliberately needed. Therefore, microalgae is a potential candidate for $\mathrm{CO}_{2}$ fixation and via its wider utilization through the application of the biomass as other value-added products such as biofuel, pharmaceutical products, food and livestock feed, it could further enhance and reinstate the natural $\mathrm{CO}_{2}$ management process.

Photosynthesis generates chemical energy from the light energy captured in the form of ATP, NADPH, and organic material whereby the chemical energy was consumed at day time and organic material was utilized without the presence of light to metabolize essential biomolecules for cell growth [4], [5]. Temperature, $\mathrm{pH}$, light and $\mathrm{CO}_{2}$ flow rate are the major factors that influence the photosynthesis activity and the behavior of microalgae growth rate [4], [5]. Among these, light is arguably one of the most important factors for microalgae growth [6]. As an endothermic reaction, carbon fixation requires energy and this is mainly provided by light. This energy enables microalgae cells to undergo the photosynthesis process which converts carbon dioxide $\left(\mathrm{CO}_{2}\right)$ into organic compounds such as carbohydrate and protein while releasing oxygen as waste. Microalgae cells cultivated under limited light conditions assimilate carbon towards the synthesis of amino acids and other essential cell constituents, but under saturated light conditions, sugars and starch are formed via the pentose phosphate-reducing pathway, suggesting the dependence of the biomass composition with the light availability [7]. In reality, cultures under continuous light are often used in research and the industry because this condition generally achieves the maximal growth rate recorded [8].

However, some findings suggest the use of light/dark cycles instead of continuous light as it allows for either an increase in final concentration or a lowering of production costs. This is because cell division occurs under dark conditions for many unicellular photosynthetic cultures while for others, cell division occurs both in the dark and the illuminated phase. For the latter, cell divisions are more frequent after the interruption of the illuminated phase. In addition, some enzymes of the pentose cycle of photosynthesis and $\mathrm{CO}_{2}$ fixation are inactive during illumination [8]. A dark phase is also necessary for the regeneration of cofactors (NAD+, NADP+) required for phase I of photosynthesis. Besides, adopting the light/dark approach is also believed to help in mitigating the adverse effects of high-intensity light exposure towards the cells [8]. Hence, it can be said that both light and dark regime are important for microalgae to achieve high productivity rate while growth and production performance are linked to the availability of light, hence highlighting the importance of adopting a light/dark cycles strategy in microalgae 
cultivation. The light duration itself might be influenced by the species of microalgae used, more specifically the environmental conditions in which they were naturally isolated [7]. For industrial applications and considering the ratio between the cost of energy and the corresponding biomass productions, 12 to 15 hours duration for the illuminated phase is generally considered as optimal for algae growth. However, feasibility of large scale application would largely depend on the cost of operation where lighting costs is definitely a significant one. This highlights the attractiveness of outdoor cultivation where light energy comes directly from the Sun. Nevertheless, discontinuous supply and varying levels of sunlight should be considered should outdoor cultivation is adopted.

In addition, light intensity must also be taken into account for an outdoor application. This is because light intensity also plays a part in influencing photosynthetic activity of a microalgae cell. Increasing illumination level would increase growth rate until a maximum level is reached. Once the threshold is reached, further increase would no longer affect cell growth and might also reduce the viability of the microalgae [9]. Hence, this paper aims to identify the most suitable light condition for outdoor microalgae cultivation, taking into account both light/dark cycles and shading configuration that will enhance cell growth and subsequently optimize the carbon fixation rate.

\section{MATERIAL AND METHODS}

\section{A. Microalgae Species}

A marine microalgae species identified as Isochrysis sp. was used throughout the study. It was previously isolated from the sea surrounding TNB Janamanjung power station, Manjung, Perak, Malaysia.

\section{B. Light Illumination Exposure Levels}

Isochrysis sp. was cultured with five different setups by varying the illumination exposure and light-dark cycle as listed Table I. In order to provide the varying levels of light exposure and to introduce a cost effective approach to it, five cylindrical columns were wrapped with black-colored plastics with different surface areas and orientation. The exposure levels were calculated based on the area which was illuminated during the course of the day. This is assumed as the total surface area of the cylindrical column, as the Sun's pathway during the day would inevitably expose all sides of the cylindrical vessel to its' light. The total light-dark cycle, on the other hand, was determined based on the assumption of the movement of one microalgae cell with regards to micro-sized air bubbles originating from aeration provided by the air pump at the bottom of the column. As the cell moves from bottom to top, it will encounter alternate light and dark exposures due to the orientation of the black wrappings. The total light-dark duration for each column was then calculated from the air flow rate and length of the black shaded area as shown in (1) below:

$$
\text { Light exposure }=\left(2 \pi r^{2}+\pi r h\right)-(L . W)
$$

with $r$ as the radius of cylindrical column, $L$ is the length of plastic, $W$ is the width of plastic and $h$ is the height of the column.

TABLE I: VARIATION OF ILLUMINATION LEVELS

\begin{tabular}{|l|l|}
\hline Column & Approximate Illumination Exposure (\%) \\
\hline A & 100 \\
\hline B & 73.6 \\
\hline C & 57.6 \\
\hline D & 29 \\
\hline E & 0 \\
\hline
\end{tabular}

\section{Culturing Conditions}

Fig. 1 shows the experimental setup of the microalgae culture. The experiment was conducted using five similar $1 \mathrm{~L}$ cylindrical columns filled with $\mathrm{f} / 2$ media, artificial sea water and microalgae inoculum. The varied illumination levels were provided through the setup mentioned in subtopic B above whereas cultivation was done outdoors under natural sunlight for 13 days. The initial optical density of the culture of all five columns was maintained at absorbance of 0.4 at $560 \mathrm{~nm}$. The aeration was provided using an air pump and maintained at the same flow rate for all columns.

\section{Data Collection and Analysis}

Samples for each columns were taken daily for the determination of microalgae growth rate. Temperature, $\mathrm{pH}$, and salinity readings were measured using PCD650 portable meter (EUTECH Instruments, Netherlands) while daily irradiation was taken using a digital lux meter (TES-1335, Taiwan). The cell growth for all samples were determined using two methods i) optical density using a spectrophotometer (HACH DR4000U, Colorado, USA) at $560 \mathrm{~nm}$; and ii) cell count measurement using a haemacytometer (Assistant, Germany).

\section{RESUlTS AND DISCUSSION}

Photosynthesis is a two-process system which includes light reactions and dark reactions. The former only occur when the cells are illuminated, and the latter, also known as carbon-fixation reactions, occur both in the presence and absence of light [8]. In overall, the cells transform light energy into chemical energy, which is stored in high-energy compounds such as ATP and NADPH. These compounds were later used in the dark phase carbon-fixation reactions to synthesize metabolic molecules essential for growth [10].

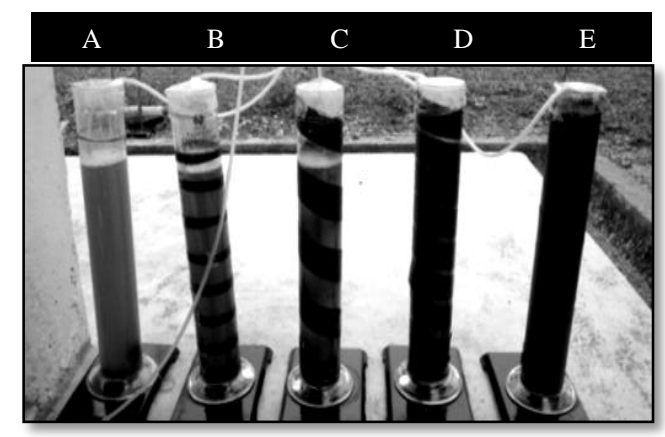

Fig. 1. Experimental setup of outdoor cultivation of Isochrysis sp. with varying illumination levels.

Hence, the growth of photosynthetic cultures such as 
microalgae is closely connected to photosynthetic activity, and by default, to carbon fixation ability of the cell. Fig. 2 (a) and (b) shows the optical density and cell count measurement of Isochrysis sp. cultured in batch condition.

In terms of growth, the results indicated that a normal growth curve was exhibited. About 3 days of lag phase growth was demonstrated for all five cultures. This might be due to the cell acclimatizing to the new environment, which differs from lab conditions especially for the case of natural sunlight that dynamically links to daily weather and sky conditions. Change of cellular physiology and biochemistry associated with photosynthesis, such as the change of pigment content in the cell, is also part of the changes that occur during light acclimatization [4]. After day 3, microalgae growth rate for columns A, B, C, and D started to increase as shown by the increases of both optical density and cell count. However, column E shows little to no growth. This is because column E was designed as such to limit its exposure to the Sun by totally covering it. This showed evidence of limited carbon source for cell growth, since the microalgae were unable to use inorganic carbon sources in the absence of light, and the organic carbon concentrations in the culture medium were insufficient for the energy maintenance of respiratory metabolism [10]. In short, when there is insufficient light, the growth of microalgae is under photolimitation condition [11].

For the other four columns, the exponential growth continued until day 7 for column A, C and D, and day 9 for column B after which stationary growth was demonstrated by all columns. Table II shows the calculated growth rates and doubling time of all five conditions. Comparing the growth rates for all columns, it is clear that column $\mathrm{B}$ which has the second highest illumination level $(73.6 \%)$ exhibited the highest growth rate $\left(\mu=0.279, t_{d}=3.6\right.$ days $)$ and a carbon fixation rate of $0.541 \mathrm{~g} \mathrm{CO}_{2} /$ day (data not published). This is followed by column $\mathrm{C}\left(\mu=0.222, t_{d}=4.5\right.$ days $)$ and $\mathrm{A}(\mu=$ $0.201, t_{d}=5.0$ days) which were exposed to $57.6 \%$ and $100 \%$ illumination, respectively. Taking into account the maximum illuminance level recorded during this cultivation period which was 42 klux compared to our current lab-scale cultivation at $2.5 \mathrm{klux}$ at publication time, it is interesting to note that the microalgae could still survive when exposed to direct sunlight. This might be due to the effect of natural dilution of light through self-shading by cells and partial shading of the column wall [12]. However, as apparent by the higher growth rate of column $\mathrm{B}$ and $\mathrm{C}$ over column A, column shading does provide some benefits to improve cell growth. Column D with $29 \%$ illumination exposure also shows good growth albeit at a lower level compared to the former three.

The amount of light received and stored by the cells has a direct relationship with the carbon-fixation capacity, consequently determining the biomass productivity and cell growth rate [7]. However the optimum level would depend on the microalgae's photosynthetic ability to fully utilize the photo-energy [9]. When the exposure corresponds to the maximum utilization ability, the efficiency of light energy conversion to biochemical energy is the highest and further increase in light levels would only cause the energy to dissipate as heat which at high enough concentration may lead to cell damage thus inhibiting the growth rate and finally causes cell death. Hence, an equilibrium have to be reached between the maximum illumination level required for maximum photosynthetic activity and the threshold limit of light intensity to prevent photoinhibition from occurring. Therefore, it can be concluded that a $70 \%$ illumination is optimum for this strain of Isochrysis sp. when cultured outdoors under natural sunlight.
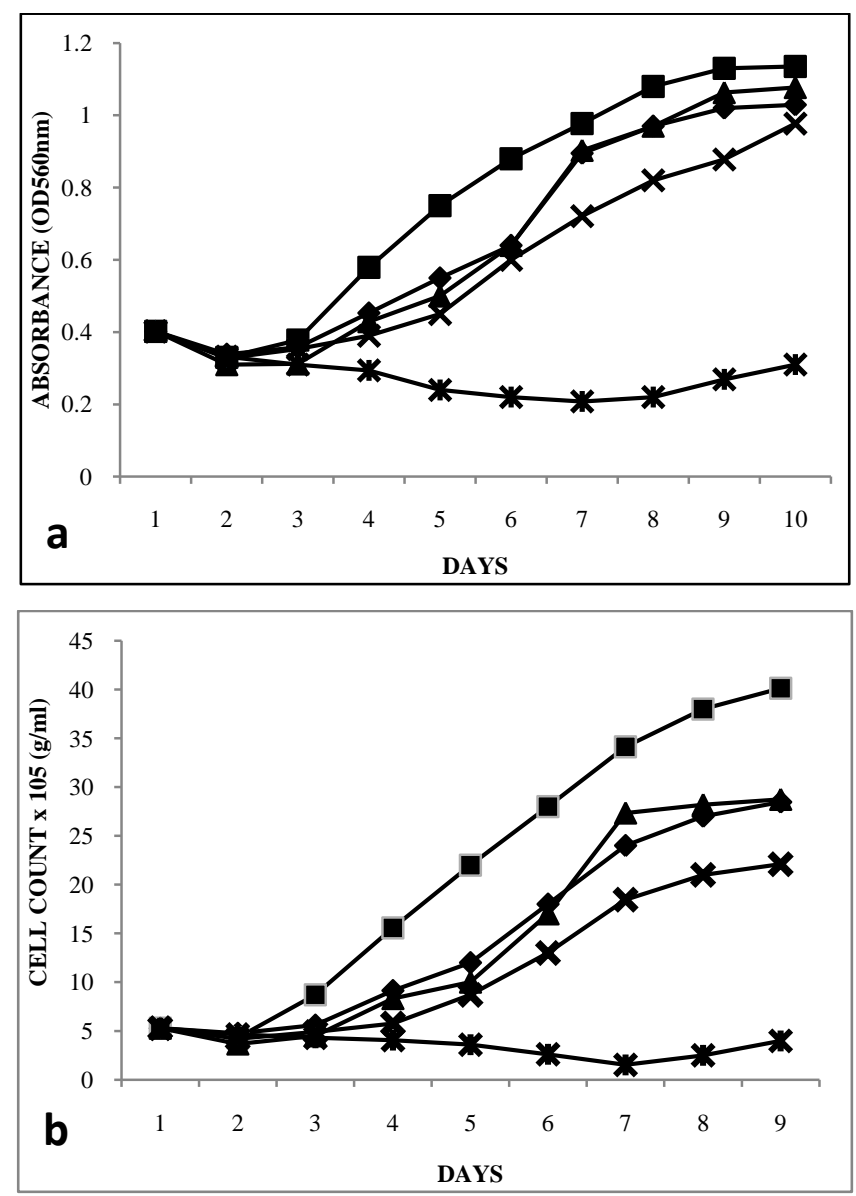

Fig. 2. Analytical results of (a) optical density measurement; (b) cell count measurement of each column. Illumination levels: $\longrightarrow 100 \%$ (Column A),

$-\mathbf{a}-73.6 \%$ (Column B), $\mathbf{\pm} 57.6 \%$ (Column C), $\rightarrow-29 \%$ (Column D) and $\rightarrow-0 \%$ (Column E).

Other than varying illumination level, the experimental setup also takes into account the light/dark cycle variation. As mentioned earlier, light/dark cycle is believed to help encounter the issue of photoinhibition which is expected to occur due to very high intensity sunlight at the outdoor cultivation location. Table III shows the calculation results of light/dark cycle for each column. From the results obtained in Fig. 2, microalgae in column B shows the highest growth rate compared to other columns. Therefore, the light/dark cycle that gives the best results was in column $\mathrm{B}$, which is 43.66-28.38 s/height of column, a medium frequency cycle (seconds-to-minutes fluctuations). A previous study by [13] mentioned that a medium frequency scale light/dark cycle is economically feasible in practice and larger photosynthetic enhancement could be expected. This is due to the 'flashing light effect' whereby photosynthesis is enhanced when the algae experience light and dark periods at high frequency [14]. The current convention is; after the capture of light energy, the photosynthetic units (PSUs) of algae cells need approximately $100 \mathrm{~ms}$ to convert it into NADPH and ATP. 
During this time, any photons reaching these 'excited' PSUs are wasted. As a result, cells exposed to flashing light with a light/dark cycle close to $100 \mathrm{~ms}$ waste less light energy than cells exposed to continuous light [4].

In outdoor cultivation, predicting the rate of photosynthesis and respiration mechanisms is challenging because algal activity is influenced by numerous factors such as light intensity, temperature, $\mathrm{pH}$, dissolved oxygen concentration, and nutrient availability [4]. Fig. 3 shows the fluctuations of both sunlight illumination and temperature within the cultivation period. On day 1 until day 3 , the weather was cloudy while the next four days showed sunny condition of which day 7 achieved the highest illumination level of 42 klux. Day 8 was cloudy while it rains on day 9 and sunny on the last day of which maximum temperature of $36^{\circ} \mathrm{C}$ was recorded. These fluctuations serve a monumental challenge in implementing microalgae outdoor cultivation for a number of reasons.

First, light intensity and temperature during the previous day affects the magnitude of night-time biomass loss as endogenous respiration rate was a function of carbohydrate content of cells [4]. Next, under light-limited conditions, the rate of photosynthesis is limited by the rate of photon supply and the rate of photon capture is considered as independent of temperature. However, in outdoor conditions, the algae are light-saturated and temperature does impact the maximum rate at which the dark reactions of photosynthesis can occur and subsequently affect the threshold for light inhibition. The rate of photosynthesis also starts to decrease with increasing light intensity due to the deactivation of key proteins in the photosynthetic units [15]. This photoinhibition phenomena occurred at different intensity levels depending on temperature conditions [8]. Previously, it was observed that light tolerance of algae was higher at high temperature which might explain our findings. Besides that, the weather conditions also play a part in microalgae growth. Cloudy weather implies that most of the light received by the cell contains mostly high-energy UV wave instead of the total sunlight spectrum [16]. Photosynthesis, on the other hand, are affected by light spectrum where green algae that has Chl $a$ chlorophyll as its major pigment, absorbs preferentially red $(\sim 650 \mathrm{~nm})$ and blue $(\sim 420 \mathrm{~nm})$ lights [9]. Hence, even if high intensity light is provided, only those within the acceptable spectrum could be utilized by the microalgae cells.

TABLE II: GROWTH RATES AND DOUBLING TIME OF ISOCHRYSIS SP. WITH VARYING ILLUMINATION LEVELS

\begin{tabular}{|l|c|c|}
\hline Column & $\begin{array}{c}\text { Maximum cell growth rate, } \mu_{\max } \\
\left(\mathrm{OD}_{560} / \text { day }\right)\end{array}$ & $\begin{array}{c}\text { Doubling time, } t_{d} \\
(\text { day })\end{array}$ \\
\hline $\mathrm{A}$ & 0.201 & 5.0 \\
\hline $\mathrm{B}$ & 0.279 & 3.6 \\
\hline $\mathrm{C}$ & 0.222 & 4.5 \\
\hline $\mathrm{D}$ & 0.195 & 5.1 \\
\hline $\mathrm{E}$ & 0.139 & 7.2 \\
\hline
\end{tabular}

TABLE III: LIGHT-DARK CYCLE FOR FIVE COLUMNS

\begin{tabular}{|l|l|}
\hline Column & Total Light-Dark Cycle \\
\hline A & $69-0$ s/height of column \\
\hline B & $43.66-28.38$ s/height of column \\
\hline C & $36.32-38.71$ s/height of column \\
\hline D & $11.41-62.44$ s/height of column \\
\hline E & $0-69$ s/height of column \\
\hline
\end{tabular}

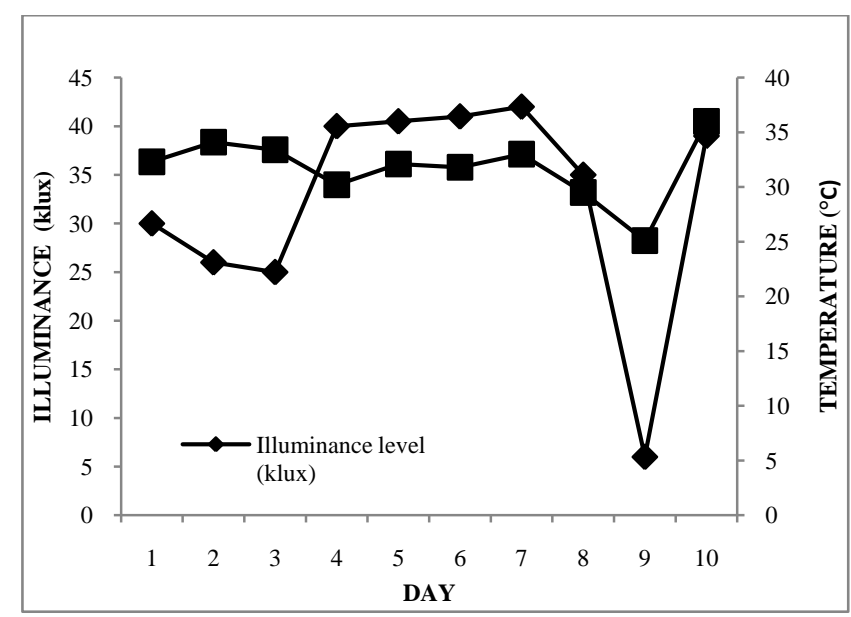

Fig. 3. Sunlight illuminance levels and temperature fluctuations during outdoor cultivation of Isochrysis sp. microalgae

\section{CONCLUSION}

From results obtained, we can conclude that column B have the highest growth rate compared to other four columns with $73.6 \%$ illumination exposure and light-dark cycle of 43.66-28.38 s/height of column. This result showed that microalgae needs both dark and light regime for an optimum photosynthetic process, cell growth rate and finally carbon fixation. This is because direct exposure from direct, intense sunlight could possibly cause cell damage while absence of light would negatively affect growth of microalgae. Thus for an optimum microalgae growth, light exposure is one of the most important parameters to be observed and optimized and light/dark cycles might help in mitigating the adverse effects of the high level of light exposure towards the cell. This result can also provide a tip in designing closed-type photobioreactors, in which certain aspects of light dilution and duration of light/dark cycle mechanism can be introduced. This can be useful for mass culture outdoor, especially in tropical conditions.

\section{ACKNOWLEDGMENT}

The authors wish to express their gratitude to TNBR Technical Board Committee and TNBR Management for the financial support for making this project a success and able to complete in time.

\section{REFERENCES}

[1] A. Bahadar and M. B. Khan, "Progress in energy from microalgae: A review," Renew. Sustain. Energy Rev., vol. 27, pp. 128-148, Nov. 2013.

[2] H. Herzog and D. Golomb, "Carbon capture and storage from fossil fuel use," Encycl. Energy, vol. I, vol. 1, pp. 1-11, 2004.

[3] F. M. Salih, "Microalgae Tolerance to High Concentrations of Carbon Dioxide: A Review,” J. Environ. Prot. (Irvine,. Calif)., vol. 02, no. 5, pp. 648-654, 2011.

[4] Q. Béchet, A. Shilton, and B. Guieysse, "Modeling the effects of light and temperature on algae growth: State of the art and critical assessment for productivity prediction during outdoor cultivation," Biotechnol. Adv., vol. 31, issue 8, pp. 1648-1663, December 2013.

[5] M. A. Qasmi, N. Raut, S. Talebi, S. A. Rajhi, and T. A. Barwani, "A Review of Effect of Light on Microalgae Growth," in Proc. World Congr. Eng., vol. I, pp. 8-10, 2012.

[6] U. Co, C. F. Knight, J. L. Milne, J. C. Cameron, L. E. Page, and S. M. Benson, "Report from Workshop on Biological Capture," pp. 1-43, 2009. 
[7] E. J. Lopes, C. H. G. Scoparo, L. M. C. F. Lacerda, and T. T. France, "Effect of light cycles (night/day) on $\mathrm{CO}_{2}$ fixation and biomass production by microalgae in photobioreactors," Chem. Eng. Process. Process Intensif., pp. 1-5, 2008.

[8] R. Bouterfas, M. Belkoura, and A. Dauta, "The effects of irradiance and photoperiod on the growth rate of three freshwater green algae isolated from a eutrophic lake source of the organisms isolated from the eutrophic Takerkoust barrage's," Lemnetica, vol. 25, no. 3, pp. 647-656, 2006.

[9] Y.-C. Jeon, C.-W. Cho, and Y.-S. Yun, "Measurement of microalgal photosynthetic activity depending on light intensity and quality," Biochem. Eng. J., vol. 27, no. 2, pp. 127-131, Dec. 2005.

[10] E. J. Lopes, C. H. G. Scoparo, L. M. C. F. Lacerda, and T. T. Franco, "Effect of light cycles (night/day) on $\mathrm{CO}_{2}$ fixation and biomass production by microalgae in photobioreactors," Chem. Eng. Process. Process Intensif., vol. 48, no. 1, pp. 306-310, Jan. 2009.

[11] S. Wahidin, A. Idris, and S. R. M. Shaleh, "The influence of light intensity and photoperiod on the growth and lipid content of microalgae Nannochloropsis sp.," Bioresour. Technol., vol. 129, pp. 7011, Feb. 2013.

[12] K. A. I. Zhang and E. Kojima, "Effect of light intensity on colony size of microalga Botryococcus braunii in bubble column photobioreactors," J. Ferment. Bioeng., vol. 86, no. 6, pp. 573-576, 1998.

[13] Y. Chen, J. Wang, W. Zhang, L. Chen, L. Gao, and T. Liu, "Forced light/dark circulation operation of open pond for microalgae cultivation," Biomass and Bioenergy, vol. 56, pp. 464-470, Sep. 2013.
[14] T. Sato, S. Usui, Y. Tsuchiya, and Y. Kondo, "Invention of outdoor closed type photobioreactor for microalgae," Energy Convers. Manag., vol. 47, no. 6, pp. 791-799, Apr. 2006.

[15] Y. Kitaya, H. Azuma, and M. Kiyota, "Effects of temperature, $\mathrm{CO}_{2} / \mathrm{O}_{2}$ concentrations and light intensity on cellular multiplication of microalgae, Euglena gracilis," Adv. Sp. Res., vol. 35, no. 9, pp. 1584-1588, Jan. 2005

[16] H. Jeong, J. Lee, and M. Cha, "Energy efficient growth control of microalgae using photobiological methods," Renew. Energy, vol. 54, pp. 161-165, Jun. 2013.

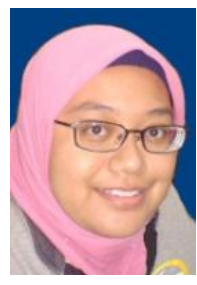

Irina Harun was born in Kuala Lumpur on August 22, 1986. She received a bachelor of engineering majoring in Biochemical Engineering from Universiti Kebangsaan Malaysia (UKM), Bangi in 2009. She went on and received her masters of Science in Chemical Engineering from the same institution in 2013. Ms. Irina had underwent two job attachments; one with Nuclear Malaysia in Dengkil, Malaysia working on irradiation effects on natural honey; and another with NanoMalaysia Berhad in Kuala Lumpur, Malaysia focusing on technological approaches and commercialization of nanotechnology in Malaysia. Currently, she is a researcher with TNB Research Sdn. Bhd located in Kajang, Malaysia since July 2013. Some of her previous research interests include biohydrogen production, nanotechnology and is currently working on carbon capture and storage technology, in particular carbon fixation by using locally isolated microalgae. 